\title{
Psyche und Rheuma
}

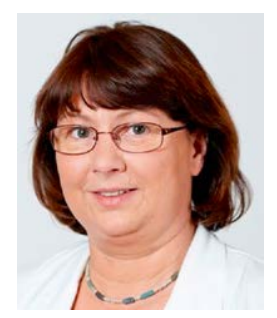

Leonore Unger

\author{
Bibliografie \\ Akt Rheumatol 2021; 46 \\ DOI 10.1055/a-1295-3881 \\ ISSN 0034-3536 \\ (c) 2021. Thieme. All rights reserved. \\ Georg Thieme Verlag KG, Rüdigerstraße 14, \\ 70469 Stuttgart, Germany \\ Korrespondenzadresse \\ Dr. Leonore Unger \\ 1. Medizinische Klinik \\ Städtisches Klinikum Dresden \\ Friedrichstraße 41 \\ 01067 Dresden \\ Leonore.Unger@klinikum-dresden.de
}

Psychische Störungen sind bei rheumatischen Erkrankungen häufig und beide beeinflussen sich gegenseitig.

In dieser Ausgabe der Aktuellen Rheumatologie versuchen wir, uns diesem komplexen Thema zu nähern. Zu Beginn haben Olga Seifert und Christoph Baerwald in einer Übersicht die wichtigsten Studien der letzte 15 Jahre zu diesem Thema zusammengefasst. Sie zeigen, wie häufig gerade Depressionen und Angststörungen bei unseren Patienten sind und welchen Einfluss sie auf den Verlauf von rheumatischen Erkrankungen haben. Gleichzeitig wird dargestellt, welchen Einfluss die Rheumaerkrankungen selbst und die in der Rheumatologie verwendeten Medikamente auf die psychische Gesundheit haben können. Bereits hier wird auf den Zusammenhang zwischen Inflammation und psychischen Veränderungen hingewiesen.

Auf diesen Aspekt geht Georg Pongratz in der nachfolgenden Arbeit genauer ein, dabei liegt der Fokus auf dem Zusammenhang zwischen psychologischem Stress und Immunsystem. Akuter und chronischer Stress wirken sich ganz unterschiedlich auf das Immunsystem aus. Die inzwischen bekannten Interaktionen zwischen Gehirn und Immunzelle, geregelt über das autonome Nervensystem und das Hormonsystem werden dargestellt.

Die Wechselwirkungen zwischen neuroendokrinem System und Autoimmunsystem bei chronisch entzündlichen Rheumaerkrankungen mit ihren vielfältigen Folgen wie Fatigue, Depression, Schlaf- und hormonellen Störungen, Mangelernährung, Knochenund Muskelabbau werden im Artikel von Florian Günther, Martin Fleck und Rainer Straub aufgezeigt. Gleichzeitig gehen die Autoren auf die Bedeutung des zirkadianen Rhythmus bei Entzündung ein und verweisen auf erste Therapiestrategien, diese Prozesse zu beeinflussen.
Christoph Schilling und Kerstin Weidner geben zum Schluss einen Überblick zum Fibromyalgiesyndrom aus psychosomatischer Perspektive. Hier findet der interessierte Leser nicht nur Daten zur Pathogenese, diagnostischen Differenzierung und Chronifizierungsfaktoren. Besonders wertvoll sind gerade für uns Rheumatologen die Hinweise für eine sinnvolle Gesprächsführung und Begleitung dieser Patienten sowie eine mögliche medikamentöse Ersttherapie.

So ist es nicht nur unser Ziel, eine entzündlich rheumatische Erkrankung früh und effizient unter Kontrolle zu bringen. Von Anfang an sollten wir die psychische Gesundheit unserer Patienten im Blick haben. In Anbetracht der langen Wartezeiten auf einen Termin beim Psychologen, Psychotherapeuten oder Psychiater müssen auch Rheumatologen Depressionen, Angsterkrankungen und somatoforme Schmerzstörungen erkennen, um eine Überdiagnostik mit iatrogener Verstärkung der Symptomatik zu vermeiden. Sehr hilfreich wäre dabei, wenn das ärztliche Gespräch als wesentlicher Aspekt der „ärztlichen Kunst“ wieder mehr in den Mittelpunkt der Wertschätzung, auch im Hinblick auf das vorhandene Zeitkontingent rücken würde. 